BIO Web of Conferences 2, 03009 (2014)

DOI: $10.1051 /$ bioconf/ 20140203009

C) Owned by the authors, published by EDP Sciences, 2014

\title{
The origin of biomolecular asymmetry - Insights from cometary and meteoritic matter
}

\author{
C. Meinert ${ }^{1}$, U.J. Meierhenrich ${ }^{1}$, L. Nahon ${ }^{2}$, S.V. Hoffmann ${ }^{3}$ and L. le Sergeant d'Hendecourt ${ }^{4}$ \\ ${ }^{1}$ Institut de Chimie de Nice, CNRS UMR 7272, Université de Nice-Sophia Antipolis, 06108 Nice \\ Cedex 2, France \\ ${ }^{2}$ Synchrotron SOLEIL, St. Aubin BP 48, 91192 Gif-sur-Yvette Cedex, France \\ ${ }^{3}$ Institute for Storage Ring Facilities, Aarhus University, 8000 Aarhus C, Denmark \\ ${ }^{4}$ Institute d'Astrophysique Spatiale, CNRS, Université Paris Sud, 91405 Orsay Cedex, France
}

\begin{abstract}
The chemistry of life is purely stereospecific. The fundamental biopolymers - proteins and nucleic acids - are intrinsically chiral due to their molecular building blocks, namely L-amino acids and D-sugars. Hypotheses for the evolutionary origin of that strict stereochemical selection include the asymmetric photochemistry model by which circularly polarized (CP) photons induced an enantiomeric excess in chiral organic molecules via asymmetric photolysis. The transfer of a distinct chirality from chiral photons to organic molecules is assumed to have occurred in environments of interstellar molecular clouds. This model is strengthened by the observation of CP light in the star-forming region of Orion. Due to our laboratory experiments mimicking cometary ice evolution, we show that molecules of prebiotic interest such as amino and diamino acids are formed in inter/protostellar environments and might have been subjected to CP vacuum-UV radiation before and during their space journey to the early Earth.
\end{abstract}

How did Life originate? The goal is to understand the scenarios by which extraterrestrial organic matter has seeded the surface of the primitive Earth and propose an astrophysical explanation for the origin of asymmetry in chiral protein and nucleotide precursors that may have led later to homochirality an assumed prerequisite for Life on Earth [1].

Why were left-handed amino acids selected for its architecture? Topical research reasons that circularly polarized light (CPL) - observed in star-forming regions - might induce an enantiomeric excess (ee) in chiral organic molecules via asymmetric interstellar photon-molecule interactions [2, 3]. A major challenge of our research is to shed some light on the asymmetric mechanism for the formation of chiral prebiotic molecules based on their interaction with UV-CPL as an external chiral force with particular emphasis on absolute asymmetric photolysis and synthesis. Therefore, we are identifying various chiral organic molecules and quantifying any small ee in carbonaceous chondrites by using the highly increased resolution power of novel multidimensional gas chromatography coupled to time-of-flight mass spectrometry (GC×GC-TOFMS) [4]. Detected ees are compared with those produced under controlled asymmetric photolysis experiments using circularly polarized synchrotron radiation (CPSR) at the synchrotron radiation source SOLEIL (France). First promising 
$\mathrm{GC} \times \mathrm{GC}$ results in terms of high photolysis rates as well as novel insights into the energydependency of asymmetric photolysis processes are currently under evaluation to accurately determine $e e$ values with reliable statistical error bars.

Chiral-specific photochemistry using CPL depends on the circular dichroism $\left(\Delta \varepsilon=\varepsilon_{\mathrm{R}}-\varepsilon_{\mathrm{L}}\right)$ of the reactants, i.e. the differences in the extinction coefficients of right- and left-handed CPL. Accordingly, circular dichroism $\Delta \varepsilon$ [5] and anisotropy spectra, $g=\Delta \varepsilon / \varepsilon$ [6], of subjected amino acids are studied in parallel. A new experimental set-up at the synchrotron radiation facility ASTRID at Aarhus University (Denmark) allowed us to record hitherto unknown anisotropy spectra of amino acids in the vacuum UV and UV spectral range [6]. These transitions are only visible in the solid amorphous state of amino acids. With the help of an ultra-high vacuum sublimation-condensationdevice we deposited amino acid enantiomers on VUV-optical windows in this particular isotropic solid state. The newly measured anisotropy spectra $g(\lambda)$ of amino acid films such as alanine (Figure 1) provide crucial information on the energy-dependent chirality transfer from light to matter. Anisotropy spectra further allow the quantitative prediction of inducible ees by asymmetric photolysis at a given extent of reaction $\xi$ using Equation 1 [6]:

$$
e e \geq\left(1-(1-\xi)^{g / 2} \times 100 \%\right.
$$

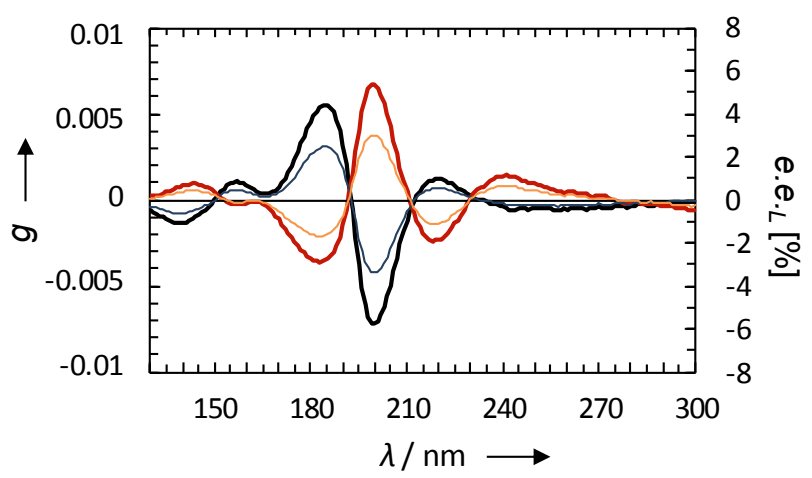

Fig. 1. Anisotropy Spectra of isotropic amorphous D-alanine (red) and L-alanine (black) in the vacuum UV and UV spectral region. Thin lines represent the L-enantiomeric excess (e.e.L) inducible by either left (orange) or right (blue) circularly polarized photons into rac-alanine at a given extent of reaction $\xi$ of $99.99 \%$.

Finally, we place our results in an astrophysical context related to the detection of $a$ ) chiral molecules in laboratory-produced cometary matter, $b$ ) the UV-CPL irradiation of interstellar ice analogs and c) ROSETTA`s enantiomerically in-situ measurements on comet 67P/ChuryumovGerasimenko. Therefore, representative interstellar molecules containing $\mathrm{C} 1$ and $\mathrm{N} 1$ units such as ${ }^{13} \mathrm{CH}_{3} \mathrm{OH}, \mathrm{NH}_{3}$ and $\mathrm{H}_{2} \mathrm{O}$ were condensed on a solid surface at $80 \mathrm{~K}$ while being irradiated at Lyman- $\alpha .20$ different amino and 6 diamino acids were identified by enantioselective GC $\times$ GCTOFMS in the simulated cometary ice residue [7]. Note, that these diamino acids (Figure 2) might have been involved in the development of a primitive genetic material, the so-called peptide nucleic acid (PNA). The PNA is a potential molecular structure from which RNA and DNA developed in distinct evolutionary processes. The results are new clues for the assumption that organic ingredients of living systems had been delivered via (micro-)meteorites and/or comets to the Early Earth from regions of the interstellar medium.

Complementary, we perform asymmetric cosmic ice evolution experiments to probe the absolute asymmetric photosynthesis of enantiomerenriched organic molecules. Here, ${ }^{13} \mathrm{C}$-labelled achiral cosmic ice mixtures are irradiated with CPSR at beamline DESIRS (SOLEIL, France). In a previous measurement campaign, the irradiation experiments produced significant ees recorded for the amino acid alanine [8]. Multidimensional gas chromatography allowed us to determine an $e e_{\mathrm{L}}$ of $-1.3 \%$ due to right-handed-CPL irradiation and $+0.7 \%$ due to left-handed-CPL irradiation. The ees were 
shown to depend on the helicity of the CPL used and are proportional to the number of photons per deposited ice molecule.

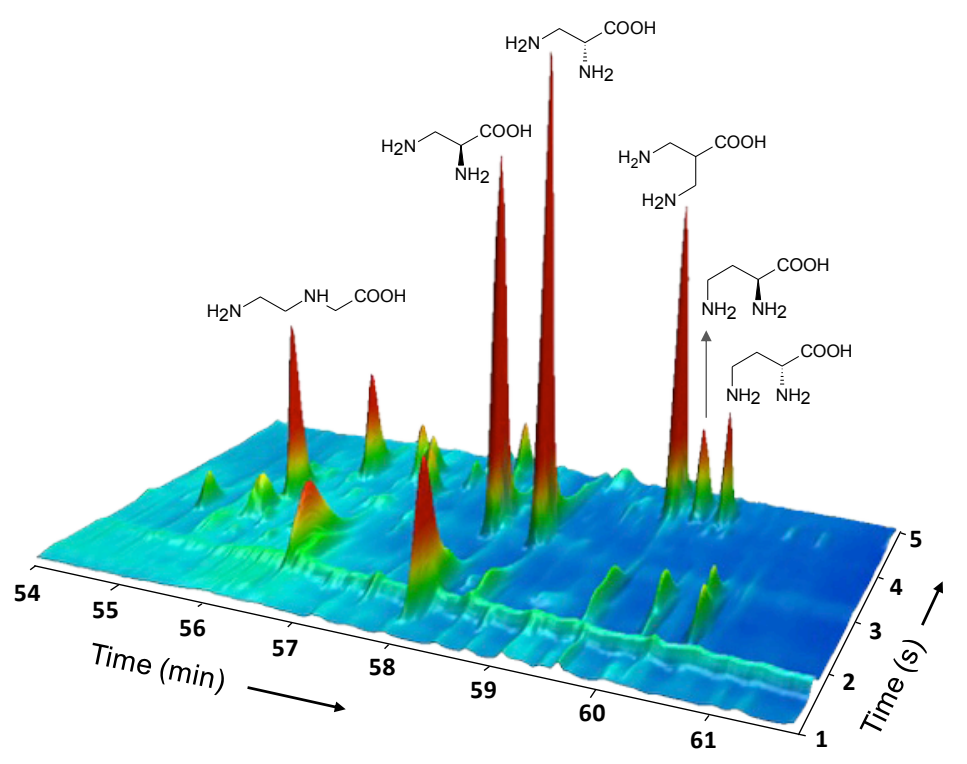

Fig. 2. Close-up view of the two-dimensional enantioselective gas chromatogram depicting ${ }^{13} \mathrm{C}$-labelled diamino acids $N$-(2-aminoethyl) glycine, L-2,3 diaminopropanoic acid (DAP), D-2,3 DAP, 3-amino-2(aminoethyl) propionic acid, L-2,4 diaminobutyric acid (DAB), and D-2,4 DAB generated under simulated interstellar pre-cometary conditions.

This research has been supported by grants from the CNRS, CNES, ANR, and the European Community`s Seventh Framework Program.

\section{References}

1. U.J. Meierhenrich, Amino Acids and the Asymmetry of Life - Caught in the Act of Formation, Springer, Heidelberg Berlin New York (2008)

2. C. Meinert, P. de Marcellus, L. Le Sergeant d'Hendecourt, L. Nahon, N.C. Jones, S.V. Hoffmann, J.H. Bredehöft, U.J. Meierhenrich, Phys. Life Rev. 8, 307-330 (2011)

3. A.C. Evans, C. Meinert, C. Giri, F. Goesmann, U.J. Meierhenrich, Chem. Soc. Rev. 41, 5447$5458(2012)$

4. C. Meinert, U.J. Meierhenrich, Angew. Chem. 124, 10610-10621 (2012); Angew. Chem. Int. Ed. 51, 10460-10470 (2012)

5. U.J. Meierhenrich, J.-J. Filippi, C. Meinert, J.H. Bredehöft, J. Takahashi, L. Nahon, N.C. Jones, S.V. Hoffmann, Angew. Chem. 122, 7966-7970 (2010); Angew. Chem. Int. Ed. 49, 7799-7802 (2010)

6. C. Meinert, J.H. Bredehöft, J.-J. Filippi, Y. Baraud, L. Nahon, F. Wien, N.C. Jones, S.V. Hoffmann, U.J. Meierhenrich, Angew. Chem. 124, 4562-4565 (2012); Angew. Chem. Int. Ed. 51, 4484-4487 (2012)

7. C. Meinert, J.-J. Filippi, P. de Marcellus, L. Le Sergeant d'Hendecourt, U.J. Meierhenrich, ChemPlusChem 77, 186-191 (2012)

8. P. de Marcellus, C. Meinert, M. Nuevo, J.-J. Filippi, G. Danger, D. Deboffle, L. Nahon, L. Le Sergeant d'Hendecourt, U.J. Meierhenrich, Astrophys. J. Lett. 727, L27 (6pp) (2011) 
